\title{
Clinical and virological properties of hepatitis $C$ virus genotype 4 infection in patients treated with different direct-acting antiviral agents
}

This article was published in the following Dove Press journal: Infection and Drug Resistance

\author{
Claudia Minosse ${ }^{1, *}$ \\ Marina Selleri',* \\ Emanuela Giombini' \\ Barbara Bartolini' \\ Maria Rosaria Capobianchi' \\ Stefano Cerilli² \\ Laura Loiacono ${ }^{2}$ \\ Chiara Taibi ${ }^{2}$ \\ Gianpiero D'Offizi \\ Fiona McPhee ${ }^{3}$ \\ AnnaRosa Garbuglia' \\ 'Department of Pre-clinical Research \\ Epidemiology and Advanced \\ Diagnostics, Laboratory of Virology, \\ National Institute for Infectious \\ Diseases "Lazzaro Spallanzani" - \\ IRCCS, Rome, Italy; ${ }^{2}$ Clinical \\ Department, Infectious Disease- \\ Hepatology Unit, National Institute \\ for Infectious Diseases, "Lazzaro \\ Spallanzani” - IRCCS, Rome, Italy; \\ "Bristol-Myers Squibb Research and \\ Development, Wallingford, CT, USA \\ *These authors contributed equally to \\ this work
}

Correspondence: Chiara Taibi Clinical Department, Infectious Disease Hepatology Unit, National Institute for Infectious Diseases “Lazzaro Spallanzani” -IRCCS, Via Portuense, 292; 00I49

Rome, Italy

Tel +390655170363

Fax +390655I70302

Email chiara.taibi@inmi.it
Background: The efficacy of direct-acting antivirals (DAAs) depends on the hepatitis $\mathrm{C}$ virus (HCV) genotype 4 (GT4) subtype which are used in the treatment of HCV. We aimed to retrospectively investigate the baseline prevalence of HCV NS5A and NS5B polymorphisms and their impact on virological outcome in GT4-infected patients treated with various DAA regimens.

Patients and methods: Available plasma samples from HCV GT4-infected patients treated with different DAA regimens were analyzed at baseline and after treatment failure, where applicable. Sanger sequencing of patient-derived NS5A and NS5B regions was performed on all available samples, while ultradeep pyrosequencing (UDPS) of NS5A and NS5B regions was performed only on samples from treatment failures at different time points.

Results: Sustained virological response (SVR) was achieved by $96 \%$ (48/50) of patients. Of 16 patients with baseline NS5A sequence, polymorphisms at amino acid positions associated with drug resistance were detected only at position 58: P58 (69.2\%) and T58 (30.8\%). Of 21 patients with baseline NS5B sequence, N142S was detected only in the two treatment failures, both with GT4d were treated with sofosbuvir (SOF)-based regimens, suggesting a potential involvement in SOF efficacy. Two patients (patient 1 [Pt1] and patient 2 [Pt2]) relapsed. In Pt1, NS5A-T56I and NS5A-Y93H/S emerged. In Pt2, NS5A-L28F emerged and a novel NS5B resistance-associated substitution (RAS), L204F, representing $1.5 \%$ of the viral population at baseline, enriched to $71 \%$ and $91.6 \%$ during and after treatment failure, respectively. UDPS of NS5B from Pt2 indicated a mixed infection of approximately 1:5, GT1a:GT4d, at baseline and GT4d during failure. Phylogenetic analysis of NS5A sequences indicated no clustering of HCV strains from patients achieving SVR vs patients who relapsed. The mean genetic distance in NS5A sequences was 5.8\%, while a lower genetic distance (3.1\%) was observed in NS5B sequences. Conclusion: Results from these analyses confirm the importance of UDPS in the analysis of viral quasispecies variability and the identification of novel RASs potentially associated with DAA treatment failure in HCV GT4-infected patients.

Keywords: hepatitis C virus genotype 4, virological failure, population sequencing, deep sequencing, resistance-associated substitution, RAS, identification

\section{Introduction}

Approximately $20 \%$ of chronic hepatitis $\mathrm{C}$ virus (HCV) infected patients worldwide are infected with genotype 4 (GT4). Its prevalence varies by geographic region, and it is the most prevalent genotype in North Africa, the Middle East, and Central and Eastern sub-Saharan Africa. Moreover, GT4 prevalence is increasing in Europe as a consequence of the migration phenomenon and transmission through injection drug 
users. ${ }^{1-4}$ GT4 is characterized by a significant genetic diversity, including at least 17 subtypes, ${ }^{5}$ of which GT subtypes $4 \mathrm{a}, 4 \mathrm{c}$, and $4 \mathrm{~d}$ are the most common subtypes in Europe. ${ }^{6,7}$ Until recently, GT4 was considered one of the more difficult genotypes to treat, at least with interferon (IFN) plus ribavirin (RBV)-based regimens. ${ }^{8,9}$ With the introduction of direct-acting antivirals (DAAs) in HCV therapy, several IFN-free options are now available for GT4-infected patients, resulting in improved efficacy and safety profiles. ${ }^{10-12}$ IFNfree options for GT4 in 2015 included sofosbuvir (SOF)/ ledipasvir (LDV), SOF/daclatasvir (DCV), or SOF/simeprevir (SMV), each with or without RBV or the combination of ombitasvir/paritaprevir/ritonavir with RBV, according to the European Association for Studies of the Liver (EASL) guidelines at that time. ${ }^{13}$

DAA efficacy varies depending on the GT4 subtype. This may be related to differences in DAA susceptibilities to certain GT4 substitutions; for example, the NS5A substitution, L28V, conferred 15-fold higher resistance to ombitasvir in vitro in GT4d than in GT4a. ${ }^{12}$ The GT4r subtype has been reported to harbor several substitutions that impair DAA potency in vitro and correlated with reduced efficacy in patients. ${ }^{14}$ However, GT4 patients are underrepresented in clinical studies for novel HCV treatments, and the number of papers describing the resistance profile of new DAAs is limited. ${ }^{12,15,16}$

In this study, the baseline prevalence of HCV NS5A and NS5B polymorphisms as well as their potential impact on virological outcome in GT4-infected patients treated with various DAA regimens was evaluated. Viral quasispecies were analyzed in patients failing treatment with the aim of monitoring viral variant dynamics potentially associated with virological failure.

\section{Patients and methods}

The institutional ethics committee of the INMI L Spallanzani Hospital approved the protocol for the described study, which was conducted in accordance with Good Clinical Practice, as defined by the principles of the Declaration of Helsinki. All patients provided written informed consent to be included in the study.

Plasma samples from 21 out of 50 GT4-infected patients treated with different DAA combination therapies at the INMI L Spallanzani Hospital in 2015 were available for this retrospective study (Table 1 ).

Representation by Caucasian patients in different study groups was determined by applying the Fisher's exact test. A $P$-value of $<0.005$ was considered significant.
HCV genotype was determined using the real-time (RT) HCV genotype II assay (Abbott Laboratories, Abbott Park, IL, USA). HCV viremia was assessed by the HCV Abbott RealTime HCV Assay (Abbott Laboratories; lower limit of detection [LOD]: $12 \mathrm{IU} / \mathrm{mL}$ ); sustained virological response (SVR) was defined as HCV RNA< LOD at week 12 post treatment. Safety and tolerability were assessed through reporting adverse events, clinical laboratory tests, and physical examinations.

\section{Extraction, amplification, and sequencing}

Viral RNA ( $60 \mu \mathrm{L}$ volume) was extracted from $600 \mu \mathrm{L}$ of plasma using the QIAsymphony DSP Virus/Pathogen Kit with an automated nucleic acid QIAsymphony instrument (Qiagen NV, Venlo, the Netherlands). The reverse transcription and first-round PCR were conducted using the OneStep RT-PCR Kit (Qiagen NV); the second-round PCR was conducted using FastStart Taq DNA Polymerase (Hoffman-La Roche Ltd., Basel, Switzerland). Positive and negative controls, which contained standardized viral RNA extracts and nuclease-free water, respectively, were included in each RT-PCR assay. The HCV NS5A and NS5B regions were amplified with nested PCR using pangenotypic primers for NS5A and NS5B, as previously described. ${ }^{17,18}$ These primer sets are summarized in Table S1. The amplicons (637 and $629 \mathrm{nt}$ for NS5A and NS5B, respectively) were sequenced directly using Prism BigDye in an ABI3100 DNA automated Sequencer (Thermo Fisher Scientific, Waltham, MA, USA).

$\mathrm{HCV}$ subtyping and NS5A and NS5B resistance analyses were performed using geno2pheno (HCV) v. 0.92. ${ }^{19}$

\section{Ultradeep pyrosequencing (UDPS) and data analyses}

Samples from two patients (patient 1 [Pt1] and patient 2 [Pt2]) who failed treatment underwent quasispecies analysis by UDPS, which was performed using the 454 Life Sciences platform (GS-junior; Hoffman-La Roche Ltd.), according to the manufacturer's instructions.

The analyses were conducted on NS5A and NS5B regions at different time points $(\mathrm{T} 0=$ baseline; $\mathrm{T} 1=8$ weeks post the end of treatment [PTWK8]; and T2= 12 weeks post the end of treatment [PTWK12]).

All reads underwent a correction pipeline with scripts developed in-house that excluded reads of $<450 \mathrm{nt}$ in length or with a mean quality score of $<20$. Moreover, the sequences were clustered with CD-HIT software, ${ }^{20}$ using a $96 \%$ identity cutoff. For the intra-sample polymorphism 
Table I Baseline characteristics of HCV GT4-infected patients

\begin{tabular}{|c|c|c|c|}
\hline Category & Total patients $(\mathrm{N}=50)$ & $\begin{array}{l}\text { Patients with analyzed } \\
\text { sequencing data }(\mathrm{N}=2 \mathrm{I})\end{array}$ & $\begin{array}{l}\text { P-value, } \\
\text { patients } \\
\text { analyzed vs } \\
\text { not analyzed } \\
\text { (Caucasian/ } \\
\text { non-Caucasian) }\end{array}$ \\
\hline Median age $\pm S D$, years (min-max) & $54 \pm 7(28-73)$ & $53 \pm 6(49-73)$ & - \\
\hline $\begin{array}{l}\text { Sex, n (\%) } \\
\text { M } \\
F \\
\end{array}$ & $\begin{array}{l}41(82) \\
9(18)\end{array}$ & $\begin{array}{l}16(76) \\
5(24)\end{array}$ & $\begin{array}{l}0.004 \\
1.000 \\
\end{array}$ \\
\hline $\begin{array}{c}\text { Ethnicity, n (\%) } \\
\text { Caucasian } \\
\text { North African }\end{array}$ & $\begin{array}{l}33(66) \\
17(34)\end{array}$ & $\begin{array}{l}19(90.5) \\
2(9.5)\end{array}$ & 0.002 \\
\hline BMI, median \pm SD $(\min -\max )$ & $25.4 \pm 2.9(19.7-32.4)$ & $25.3 \pm 2.7(19.7-30.9)$ & - \\
\hline HCV viral load (log), median (min-max) & $5.8(2.4-6.6)$ & $5.9(3.0-6.6)$ & - \\
\hline $\mathrm{HIV}+, \mathrm{n}(\%)$ & $7(14)$ & $5(24)$ & 0.286 \\
\hline $\begin{array}{l}\text { Previous treatment, } \mathrm{n}(\%) \\
\text { Naïve } \\
\text { Experienced } \\
\text { Relapser } \\
\text { Nonresponder } \\
\text { Not evaluable } \\
\end{array}$ & $\begin{array}{l}23(46) \\
27(54) \\
9(33.3) \\
13^{a}(48.2) \\
5(18.5) \\
\end{array}$ & $\begin{array}{l}\text { I0 (47.6) } \\
\text { II }(52.4) \\
\text { I }(9.1) \\
7^{\mathrm{a}}(63.6) \\
3(27.3) \\
\end{array}$ & $\begin{array}{l}0.339 \\
0.005 \\
1.000 \\
0.029 \\
0.100 \\
\end{array}$ \\
\hline $\begin{array}{l}\text { Fibrosis stage, } \mathrm{n}(\%) \\
\text { FI-F2 } \\
\text { F3 } \\
\text { F4 } \\
\text { Child-Pugh A } \\
\text { Child-Pugh B }\end{array}$ & $\begin{array}{l}3(6) \\
15(30) \\
32(64) \\
24(75) \\
8(25)\end{array}$ & $\begin{array}{l}2(9.5) \\
8(38.1) \\
\text { II (52.4) } \\
7(63.6) \\
4(36.4)\end{array}$ & $\begin{array}{l}0.333 \\
1.000 \\
0.002 \\
1.000 \\
0.429\end{array}$ \\
\hline $\begin{array}{l}\text { Antiviral regimens, n (\%) } \\
\text { SOF + RBV until OLT } \\
\text { SOF + SMV + RBV I } 2 \text { wks } \\
\text { SOF + SMV I } 2 \text { wks } \\
\text { SOF + DCV + RBV I } 2 \text { wks } \\
\text { SOF + DCV } 24 \text { wks } \\
\text { OMV/PTV/ritonavir + RBV } 12 \text { wks } \\
\text { OMV/PTV/ritonavir + RBV } 24 \text { wks } \\
\text { SOF/LDV I } 2 \text { wks } \\
\text { SOF/LDV + RBV I2 wks } \\
\text { SOF/LDV } 24 \text { wks } \\
\text { SOF/LDV + RBV } 24 \text { wks }\end{array}$ & $\begin{array}{l}2(4) \\
9(18) \\
6(12) \\
2(4) \\
3(6) \\
9(18) \\
5(10) \\
2(4) \\
4(8) \\
5(10) \\
3(6)\end{array}$ & $\begin{array}{l}2(9.5) \\
4(19.1) \\
2(9.5) \\
0 \\
1(4.8) \\
5(23.8) \\
4(19.1) \\
0 \\
0 \\
2(9.5) \\
1(4.8)\end{array}$ & - \\
\hline
\end{tabular}

Note: a One patient was treated with DCV + pIFN + RBV.

Abbreviations: BMI, body mass index; DCV, daclatasvir; F, female; GT4, genotype 4; HCV, hepatitis C virus; LDV, ledipasvir; M, male; max, maximum; min, minimum; OLT, orthotopic liver transplantation; OMV, ombitasvir; pIFN, peg-interferon; PTV, paritaprevir; RBV, ribavirin; SOF, sofosbuvir; SMV, simeprevir; wks, weeks.

analysis, a threshold of $1 \%$ was applied as described previously. ${ }^{21}$

\section{Phylogenetic analysis}

The CLUSTAL W program was used for multiple sequence alignments. All sequences were trimmed to include the largest common region encompassing amino acid positions 1-147 in NS5A and 146-340 in NS5B and compared with a
GT4d reference sequence (accession number: DQ418786), as described by Smith et al. ${ }^{22}$

Phylogenetic trees were built using study patient sequences as well as HCV GT4 sequences from the Los Alamos database, for which geographic location was reported. The best-fit model $(\mathrm{K} 2+\mathrm{G}+\mathrm{I})$ was selected according to the akaike information criterion, and trees were reconstructed using the maximum likelihood method implemented in 
MEGA6. ${ }^{23}$ Bootstrap values were calculated using 500 bootstrap iterations; values of $>70 \%$ were considered significant.

The inter-patient nucleotide distance was calculated using MEGA6 software and expressed as the number of base substitutions per site between sequences.

\section{Results}

\section{Baseline characteristics}

Among 50 GT4 patients treated with different DAA combination therapies at the INMI L Spallanzani Hospital in 2015, 21 were selected for this study based on the availability of baseline samples. Demographic and virological characteristics of these patients are summarized in Table 1 .

Baseline characteristics of the $50 \mathrm{HCV}$ GT4-infected patients are summarized in Table 1 . Median age was 54 years (range: 28-73); 17 (34\%) patients were North Africans and 33 (66\%) were Caucasians. The median baseline HCV RNA viremia for all patients was $5.8 \log 10 \mathrm{IU} / \mathrm{mL}$ (range 2.4-6.6); seven patients $(14 \%)$ were HIV coinfected. With respect to fibrosis stage, three patients (6\%) had F1-F2, $15(30 \%)$ had advanced fibrosis (F3), and 32 (64\%) were cirrhotic (75\% Child-Pugh A and $25 \%$ Child-Pugh B). Almost half (46\%) of the patients were treatment naïve, and 54\% were treatment experienced. All treatment-experienced patients had pIFN + RBV failures (Table 1) except one who failed DCV+ pIFN + RBV; this patient achieved SVR when retreated with an IFN-free regimen.

Furthermore, Caucasians were overrepresented in the fibrosis stage F4 patient group with analyzed sequencing data $(\mathrm{n}=21$; Table 1$)$.

Patients were treated according to the EASL guidelines applicable at the start of treatment: the antiviral regimens are summarized in Table 1.

After 4 weeks of treatment, $40 \%$ of patients (20/50) had undetectable HCV RNA; at the end of treatment, all patients had HCV RNA that was either undetectable or below the limit of quantification.

All regimes were safe and well tolerated. Adverse events were mild (grade 1 according to Common Terminology Criteria for Adverse Events, CTCAE) $:{ }^{24}$ headache, asthenia, fatigue, and nausea were the most commonly reported.

Almost all patients (96\%) achieved SVR. Two patients relapsed at PTWK4. Pt1 was a HCV mono-infected, cirrhotic (Child-Pugh A, MELD10) patient who was treated with $\mathrm{SOF} / \mathrm{LDV}$ for 24 weeks; Pt 2 was $\mathrm{HCV} / \mathrm{HIV}$ coinfected with decompensated cirrhosis (Child-Pugh B, MELD16) who had received SOF/DCV for 24 weeks.

Of the 21 patient-derived baseline samples sequenced, HCV NS5A and NS5B data were obtained from 16 and 21 patients, respectively; no relevant substitutions at positions known to be associated with drug resistance were detected in either gene by Sanger sequencing. At the NS5A polymorphic position 58, $\mathrm{T}$ and $\mathrm{P}$ were observed in $30.8 \%$ and $69.2 \%$ of cases, respectively. Sanger sequencing of treatment failure samples from the two patients who relapsed showed emergence of the most described NS5A resistance-associated substitution (RAS), Y93H/S, in Pt1, while Pt2 had emergent NS5A-L28F and NS5B-L204F (a novel RAS) at both time points sampled after failure (PTWK8 and PTWK12).

UDPS was performed on samples from Pt1 and Pt2 at baseline, PTWK8, and PTWK12. After pipeline correction of UDPS raw data, a median of 1,725 (range: 659-1,754) and 769 (range: 330-4,581) reads were obtained for the NS5A and NS5B sequences, respectively. Polymorphisms detected by UDPS are summarized in Table $2 \mathrm{~A}$ and $\mathrm{B}$.

Concerning NS5A (Table 2A), longitudinal UDPS data were obtained only for Pt1. Y93H was absent at T0, appeared at PTWK8 and persisted at PTWK12 as the predominant variant (77.7\% and $62.2 \%$, respectively). Another variant at the same position, Y93S (RAS previously observed in patients infected with HCV GT1a and GT1b), was observed at PTWK8 and PTWK12 with lower frequency (PTWK8=21.4\% and PTWK12=34.9\%). The NS5A RAS L28V was also detected as a minority variant (3.2\%) at PTWK12. Furthermore, UDPS confirmed the presence of other substitutions not commonly described as NS5A RASs: T56I (previously described to confer DCV resistance when associated with R30Q-Q54H-A92K in GT1b) ${ }^{25}$ was absent at baseline, but was detected as a predominant variant after failure (76.4\% at PTWK8 and $62.1 \%$ at PTWK12); I101T, present as minority variant at baseline (1.6\%), was elevated at PTWK8 (16.3\%) and PTWK12 (29.3\%).

Regarding NS5B (Table 2B), longitudinal UDPS data were obtained for both Pt1 and Pt2, although PTWK8 data were not available for Pt1. In both patients, the frequency of N142S fluctuated around $20-30 \%$ at all tested time points (ie, at baseline and after failure). Of note, the frequency of L204F enriched from $1.5 \%$ at baseline to $77.0 \%$ at TPWK8 and $91.6 \%$ at PTWK12 in Pt2. Furthermore, polymorphisms were observed at positions 231, 270, and 276 in both patients but with a stable frequency at baseline, PTWK8, and PTWK12. These polymorphisms were also present in patients who achieved SVR (data not shown).

Interestingly, HCV genotyping of the baseline sample from Pt2, using the RT HCV genotype II assay indicated a GT1a/ GT4d mixed infection. Sanger sequencing of the baseline sample classified Pt2 as GT1 a based on NS5A and GT4d based on NS5B. At the post-baseline time points, $\mathrm{Pt} 2$ was classified as 
Table 2 Frequency of HCV NS5A (A) and NS5B (B) amino acid substitutions detected by UDPS in treatment failures when compared with the reference DQ4I8786 strain

\begin{tabular}{|c|c|c|c|c|c|}
\hline \multicolumn{4}{|l|}{ A } & & \\
\hline \multirow[b]{2}{*}{ NS5A substitution } & \multicolumn{3}{|l|}{ PtI } & & \\
\hline & T0 (reads) & TI (reads) & T2 (reads) & & \\
\hline VI2I & $--^{\mathrm{a}}$ & $--^{a}$ & $1.2 \%(339)$ & & \\
\hline T2IA & - & - & $1.2 \%(339)$ & & \\
\hline L28V & - & - & $3.2 \%(339)$ & & \\
\hline M3IL & $7.4 \%(867)$ & - & - & & \\
\hline V34I & $99.2 \%(867)$ & $99.5 \%(87 I)$ & $99.1 \%(339)$ & & \\
\hline F37L & $2.9 \%(867)$ & - & - & & \\
\hline R44K & $8.7 \%(867)$ & - & - & & \\
\hline M53V & $1.0 \%(867)$ & - & - & & \\
\hline M53T & - & - & $1.2 \%(339)$ & & \\
\hline $\mathrm{H} 54 \mathrm{R}$ & - & $\mathrm{I} .3 \%(87 \mathrm{I})$ & - & & \\
\hline T561 & - & $76.1 \%(1,754)$ & $62.1 \%(659)$ & & \\
\hline T58S & - & $3.1 \%(1,754)$ & $2.4 \%(659)$ & & \\
\hline T58A & - & - & $1.7 \%(659)$ & & \\
\hline L63R & $4.3 \%(1,725)$ & - & - & & \\
\hline V67I & $2.3 \%(I, 725)$ & - & - & & \\
\hline $\mathrm{N} 69 \mathrm{~K}$ & - & - & $1.8 \%(659)$ & & \\
\hline H85R & $6.1 \%(1,725)$ & - & - & & \\
\hline T87A & $6.3 \%(I, 725)$ & - & - & & \\
\hline $\mathrm{Y93H}$ & - & $77.7 \%(1,754)$ & $62.2 \%(659)$ & & \\
\hline Y93S & - & $21.4 \%(1,754)$ & $34.9 \%(659)$ & & \\
\hline IIOIT & $\mathrm{I} .6 \%(\mathrm{I}, 725)$ & $16.3 \%(1,754)$ & $29.3 \%(659)$ & & \\
\hline IIOIV & $67.1 \%(1,725)$ & $78.8 \%(1,754)$ & $62.2 \%(659)$ & & \\
\hline PI04F & - & - & $3.8 \%(659)$ & & \\
\hline PI04L & - & - & $30.2 \%(659)$ & & \\
\hline DI05N & $52.7 \%(1,725)$ & $78.7 \%(1,754)$ & $64.3 \%(659)$ & & \\
\hline VI08F & $97.9 \%(172)$ & $99.5 \%(1,754)$ & $98.6 \%(659)$ & & \\
\hline $\mathrm{R} / 23 \mathrm{G}$ & $1.5 \%(1,725)$ & - & - & & \\
\hline VI24M & $\mathrm{I} .68 \%(\mathrm{I}, 725)$ & - & - & & \\
\hline $\mathrm{DI} 26 \mathrm{G}$ & - & - & I.7\% (659) & & \\
\hline $\mathrm{D} / 26 \mathrm{~N}$ & $1.6 \%(1,725)$ & - & - & & \\
\hline DI26S & - & - & $3.5 \%(659)$ & & \\
\hline VI30I & $99.8 \%(1,725)$ & $98.2 \%(1,754)$ & $99.6 \%(659)$ & & \\
\hline GI55S & - & $1.1 \%(883)$ & - & & \\
\hline $\mathrm{VI} 56 \mathrm{I}$ & $\mathrm{I} .3 \%(858)$ & - & - & & \\
\hline RI57K & - & $7.1 \%(883)$ & $7.5 \%(320)$ & & \\
\hline HI6IY & - & - & $1.3 \%(320)$ & & \\
\hline LI68M & $99.5 \%(858)$ & $99.8 \%(883)$ & $99.6 \%(320)$ & & \\
\hline DI7IE & $100 \%(858)$ & $99.4 \%(883)$ & $99.4 \%(320)$ & & \\
\hline EI72G & - & - & $1.3 \%(320)$ & & \\
\hline TI74S & $86.4 \%(858)$ & $4 I .8 \%(883)$ & $32.4 \%(320)$ & & \\
\hline SI76T & $99.8 \%(858)$ & $99.9 \%(883)$ & $48.0 \%(320)$ & & \\
\hline TI8IN & $13.3 \%(858)$ & $15.5 \%(883)$ & $31.9 \%(320)$ & & \\
\hline VI83L & $3.7 \%(858)$ & - & - & & \\
\hline $\mathrm{SI} 86 \mathrm{P}$ & $1.4 \%(858)$ & - & - & & \\
\hline \multicolumn{6}{|l|}{ B } \\
\hline & PtI & & Pt2 & & \\
\hline NS5B substitution & T0 (reads) & T2 (reads) & T0 (reads) & TI (reads) & T2 (reads) \\
\hline $1138 \mathrm{~F}$ & - & - & - & $2.0 \%(249)$ & - \\
\hline $\mathrm{NI} 42 \mathrm{~S}$ & $23.4 \%(1,557)$ & $30.1 \%(3,4 \mid 7)$ & $32.2 \%(17 I)$ & $25.7 \%$ (249) & $24.8 \%(396)$ \\
\hline VI44A & - & - & $1.2 \%(I 7 I)$ & - & - \\
\hline FI45L & - & - & - & - & $1.0 \%(396)$ \\
\hline $\mathrm{SI} 46 \mathrm{P}$ & - & - & - & - & $1.0 \%(396)$ \\
\hline
\end{tabular}


Table 2 (Continued)

\begin{tabular}{|c|c|c|c|c|c|}
\hline \multicolumn{6}{|l|}{ B } \\
\hline & PtI & & Pt2 & & \\
\hline NS5B substitution & T0 (reads) & T2 (reads) & T0 (reads) & TI (reads) & T2 (reads) \\
\hline KI48E & - & - & - & - & $1.0 \%(396)$ \\
\hline EI50G & - & - & $1.2 \%(I 7 I)$ & - & - \\
\hline KI5IR & $\mathrm{I} .3 \%(\mathrm{I}, 557)$ & - & - & - & - \\
\hline GI53S & - & - & - & $1.6 \%(249)$ & - \\
\hline LI65R & - & - & $1.2 \%(I 7 I)$ & - & - \\
\hline $\mathrm{CI} 70 \mathrm{R}$ & - & - & $1.2 \%(I 7 I)$ & - & - \\
\hline GI88E & - & - & - & - & $1.3 \%(396)$ \\
\hline E202D & $1.0 \%(1,988)$ & - & - & - & - \\
\hline F203L & $1.3 \%(1,988)$ & - & - & - & - \\
\hline L204F & - & - & $1.5 \%(330)$ & $77.0 \%(522)$ & $91.6 \%(769)$ \\
\hline L204P & - & - & $2.1 \%(330)$ & - & - \\
\hline L204R & - & - & $3.9 \%(330)$ & - & - \\
\hline L204V & - & - & - & $19.7 \%(522)$ & - \\
\hline L205F & - & - & $2.7 \%(330)$ & $20.9 \%(522)$ & - \\
\hline N206S & $14.6 \%(1,988)$ & $2.8 \%(4,58 \mathrm{I})$ & - & $1.5 \%(522)$ & - \\
\hline W208R & - & I.I\% $(4,58 \mathrm{I})$ & - & - & - \\
\hline $\mathrm{K} 2 \mathrm{I} 2 \mathrm{R}$ & $1.1 \%(1,988)$ & - & - & - & - \\
\hline T213A & $1.1 \%(1,988)$ & I.0\% (4,58I) & - & - & - \\
\hline $\mathrm{T} 213 \mathrm{~N}$ & - & - & - & $4.6 \%(522)$ & - \\
\hline M2I5I & - & - & - & - & I5.1\% (769) \\
\hline M2I5T & - & $2.0 \%(4,58 \mathrm{I})$ & - & - & $\mathrm{I} .4 \%(769)$ \\
\hline S218P & - & $1.5 \%(4,58 \mathrm{I})$ & - & - & - \\
\hline K23IR & $99.5 \%(1,988)$ & $99.7 \%(4,58 I)$ & $98.5 \%(330)$ & $100 \%(522)$ & $99.5 \%(769)$ \\
\hline P247L & - & - & - & - & I.4\% (769) \\
\hline S254T & - & - & $1.2 \%(330)$ & - & - \\
\hline S269G & I.2\% $(1,988)$ & - & - & - & - \\
\hline K270R & $99.4 \%(1,988)$ & $99.4 \%(4,58 \mathrm{I})$ & $99.4 \%(330)$ & $99.6 \%(522)$ & $99.6 \%(769)$ \\
\hline $\mathrm{D} 272 \mathrm{~N}$ & $\mathrm{I} .1 \%(\mathrm{I}, 988)$ & - & - & - & - \\
\hline $1276 \mathrm{~T}$ & $99.7 \%(1,988)$ & $99.6 \%(4,58 I)$ & $99.4 \%(330)$ & $99.4 \%(522)$ & $99.9 \%(769)$ \\
\hline G283S & - & - & - & - & I.0\% (769) \\
\hline F289L & - & I.2\% $(4,58 \mathrm{I})$ & - & - & - \\
\hline S300T & - & - & $3.3 \%(330)$ & $10.3 \%(522)$ & $7.4 \%(769)$ \\
\hline I303V & - & $\mathrm{I} .5 \%(4,58 \mathrm{I})$ & - & - & - \\
\hline I303T & - & $2.5 \%(4,58 \mathrm{I})$ & $1.8 \%(330)$ & - & - \\
\hline G307S & $4.2 \%(43 I)$ & - & - & - & - \\
\hline K309R & $1.6 \%(43 I)$ & - & - & - & - \\
\hline D3I0N & - & $7.1 \%(1,165)$ & $4.4 \%(159)$ & - & - \\
\hline D3I0G & - & - & $1.3 \%(159)$ & - & - \\
\hline M3I3V & $1.2 \%(43 I)$ & - & - & - & - \\
\hline $\mathrm{D} 327 \mathrm{~N}$ & - & $1.1 \%(1,165)$ & - & - & - \\
\hline G328S & $\mathrm{I} .2 \%(43 \mathrm{I})$ & - & - & - & \\
\hline E33ID & $1.2 \%(43 I)$ & - & - & - & - \\
\hline E33IT & & $35.1 \%(1,165)$ & & & \\
\hline
\end{tabular}

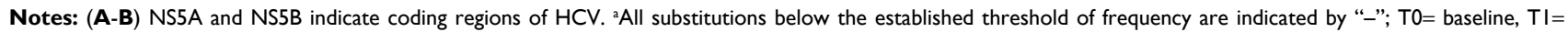
PTWK8, and T2= PTWK 12 .

Abbreviations: $\mathrm{HCV}$, hepatitis C virus; PtI, patient I; Pt2, patient 2; PTWK8, 8 weeks post the end of treatment; PTWK12, 12 weeks post the end of treatment; UDPS, ultradeep pyrosequencing.

GT4d based on both NS5A and NS5B Sanger sequences. UDPS analysis of the NS5B region resolved this apparent discrepancy. In fact, UDPS analysis of NS5B confirmed the concomitant presence of GT1a and GT4d sequences at baseline (ratio 1:5) and disappearance of GT1a after failure. As summarized in Table 2, the genetic distance decreased after treatment failure along with the emergence of RAS-bearing variants, consistent with drug resistance selection exerted by therapy.

\section{Phylogenetic analysis}

Figure 1 depicts the phylogenetic trees for the available patient-derived NS5A and NS5B Sanger sequences. The 
analysis confirmed the geno2pheno subtyping results: all sequences clustered around GT4d except for two (Pt5 and Pt9: GT4a). In addition, at baseline, Pt2 clustered with GT1a for NS5A and with GT4d for NS5B; at subsequent time points, the sequences of both regions clustered with GT4d, in agreement with UDPS data (as described earlier).

Phylogenetic analysis based on NS5A showed that the sequences from study patients (SVR and treatment failures) did not form a separate cluster within GT4d sequences; in fact, the mean genetic distance among study patients was $5.8 \%$, ie, very similar to the distance vs all other GT4d sequences in the analysis (5.9\%). In addition, the mean genetic distance vs sequences from Italian and French patients from other studies was $6.7 \%$, and $5.4 \%$, respectively.

Genetic distance between the NS5B sequences was lower: the distance between study patients was $3.1 \%$, which was similar to the distance vs all other GT4d sequences in the analysis (3.8\%). In addition, the mean genetic distance vs sequences from Italian and French patients from other studies was $3.7 \%$ and $3.9 \%$, respectively.

Of note for the two patients who failed treatment, the intra-patient genetic distance for NS5A was $1.7 \%$ in Pt1, and for NS5B, it was $1.4 \%$ in both Pt1 and Pt2.

Sequences from study patients (including two treatment failures sampled at multiple time points) are shown in red (NS5A: 16 patients, NS5B: 21 patients). Bootstrap analysis with 500 replicates was performed to assess the significance of the nodes; values $>70 \%$ are considered significant. The bar represents substitution per nucleotide position. Reference sequences are labeled by genotype or subtype (when available), their accession numbers, and their country of origin: AU, Australia; BE, Belgium; CA, Canada; CY, Cyprus; CN, China; EG, Egypt; ES, Spain; FR, France; GB, Great Britain; IT, Italy; JP, Japan; PT, Portugal; US, United States.

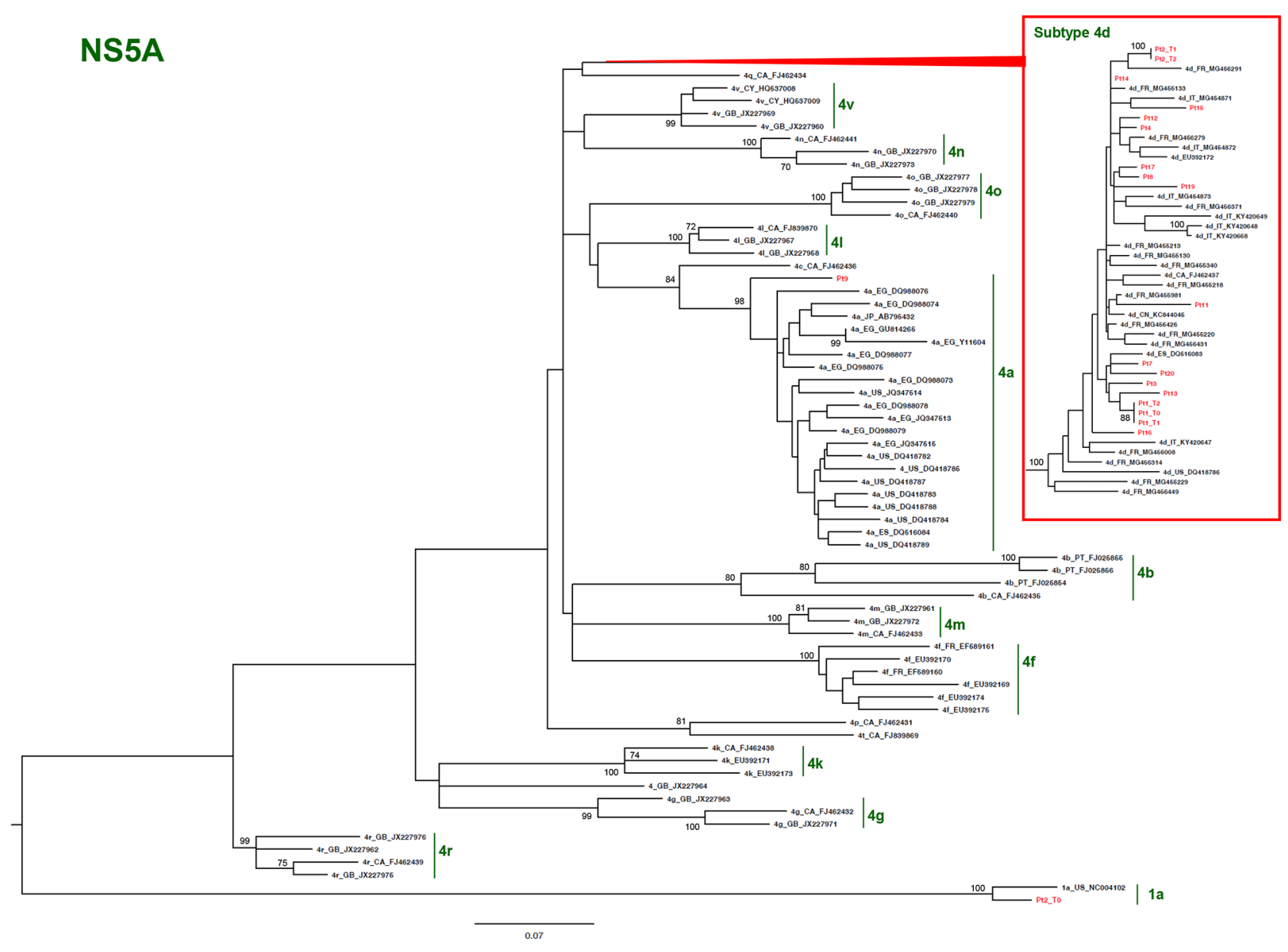

Figure I (Continued) 


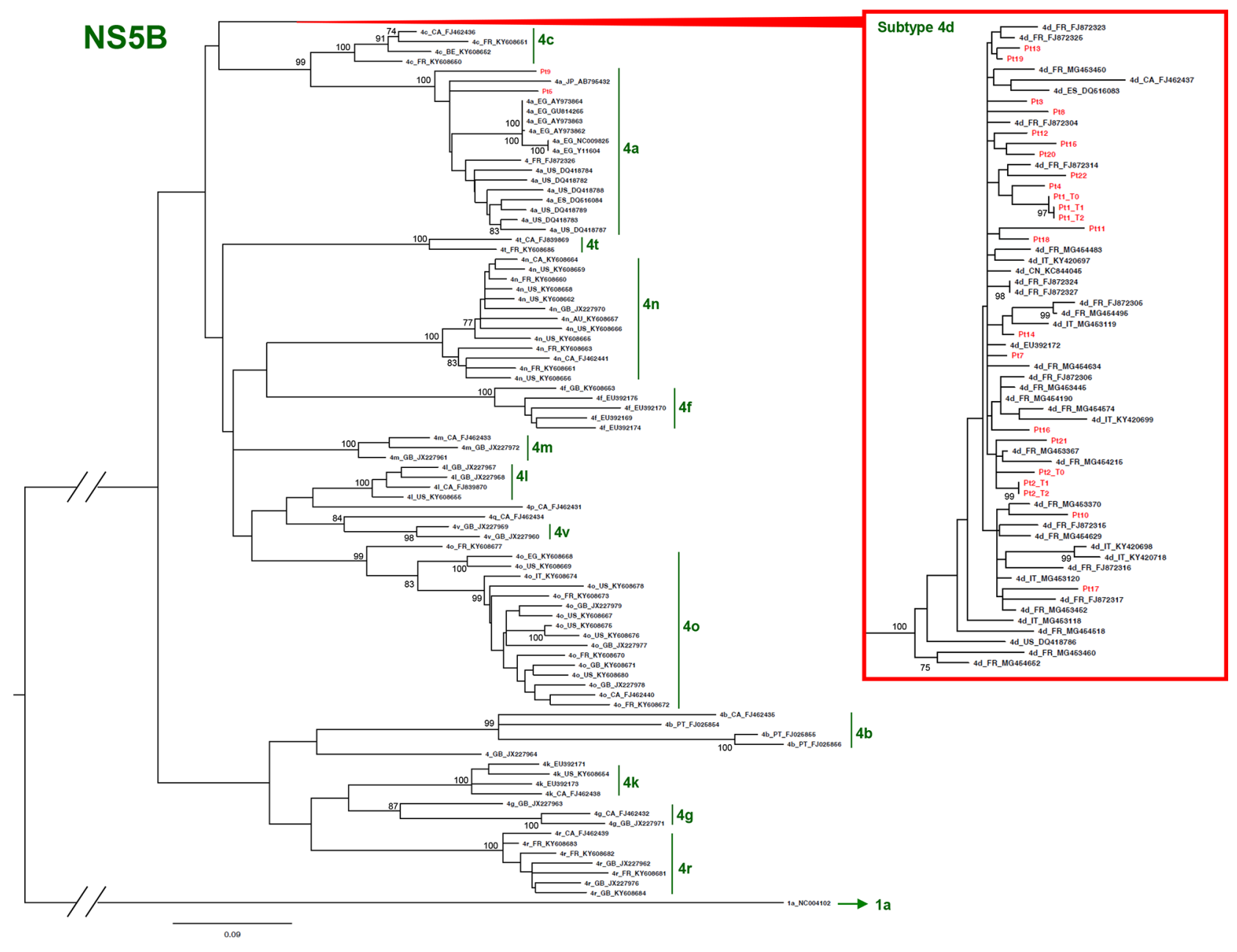

Figure I Phylogenetic trees based on HCV NS5A and NS5B Sanger sequences. Abbreviation: $\mathrm{HCV}$, hepatitis $\mathrm{C}$ virus.

\section{Discussion}

In this study, we assessed the variability of HCV GT4 subtypes in patients treated with DAA-based therapies at the INMI L Spallanzani Hospital in Italy during 2015. As expected, GT4d was the predominant subtype observed among the study population (19/21 patients, 90.5\%). Only two patients were infected with HCV GT4a.

Analyses of patient-derived baseline HCV NS5A and NS5B sequences that indicated minimal genetic variability at any amino acid position were reported to be associated with drug resistance. Preexisting RASs were infrequently observed in the NS5A region: T58P was the most frequently observed polymorphism (69.2\%); however, none of the patients with this polymorphism experienced virological failure. Although polymorphisms at this position have been associated with virological failure, T58P has not been associated with virological failure.
UDPS was performed on two patients who failed treatment. By this approach, the pattern of substitutions identified by Sanger sequencing was confirmed; in addition, a number of minor variants could be evidenced, and quantitative changes could be evaluated.

The only previously reported GT4 NS5A RAS observed in Pt1 was Y93H (PTWK8=77.7\%; PTWK12=62.2\%), which was absent at baseline (by both Sanger and UDPS), in agreement with the dynamics previously reported in a GT4 French cohort undergoing different DAA regimens ${ }^{26}$ and previously reported in GT1 and GT3 patients treated with SOF + DCV. ${ }^{27-31}$

Moreover, the coexisting NS5A-Y93S minority substitution was evidenced by UDPS: it emerged at PTWK8 (21.4\%) and was enriched at PTWK12 (34.9\%), suggesting a potential immunological pressure in this region. Previous studies conducted in vitro in GT1a have shown that Y93S may 
reduce DCV activity; however, its influence in GT4d subtype is unknown ${ }^{16,32}$ although the level of resistance conferred by Y93S on DCV (or LDV) was comparable with $\mathrm{Y} 93 \mathrm{H}$ in an in vitro GT4a NS5A hybrid replication assay.

A novel HCV GT4 NS5A substitution (T56I) enriched at virological failure, suggesting a possible role in conferring drug resistance. NS5A-T56I has emerged in HCV GT1b patients failing treatment with DCV+ IFN + RBV (Table 2). ${ }^{25}$ The T56I substitution alone conferred no resistance to DCV when tested in vitro, although an additional fourfold reduction in resistance was reported with certain combinations of other NS5A substitutions. This substitution may therefore play a secondary role in LDV resistance as well.

The NS5B substitution, N142S, was detected in both HCV GT4d treatment failures. Previous reports showed that this substitution emerged in HCV GT1b in in vitro studies, although its association with SOF resistance in the clinic has not been determined. This substitution fluctuated around $20-30 \%$ from baseline to post-failure in both virological failures. Although these findings are from only two patients, it may suggest, at least in GT4d, that the lack of enrichment, along with virological failure, may indicate independency from SOF pressure; more studies are necessary to substantiate this point.

In Pt2, the NS5B substitution, L204F, was detected by UDPS as a minority variant (1.5\%) at baseline, which enriched to $77.0 \%$ at PTWK8 and to $91.6 \%$ at PTWK12. To the best of our knowledge, this substitution has never been associated with NS5B inhibitor-based regimen failure in vivo; further investigation is required to assess whether this substitution interferes with SOF efficacy/activity and whether it is specific to GT4d.

Furthermore, the quasispecies composition identified by using UDPS of NS5B from the patient classified as having a mixed infection of GT1a and GT4 with a conventional genotyping method explained the apparently conflicting results based on Sanger sequencing. In fact, UDPS of NS5B revealed the presence of two distinct elements of viral quasispecies, with GT1a representing the minor component, possibly susceptible to the DAA combination used for treatment since it was not detected in post-failure samples. Interestingly, both treatment failure patients were cirrhotic (Pt1, Child-Pugh A and Pt2, Child-Pugh B); this is in agreement with findings from other studies, where cirrhotic status impacted therapy success in patients infected with other HCV genotypes. ${ }^{33}$

Overall, these findings confirm the importance of UDPS in the analysis of quasispecies variability and suggest a potential role in updating the list of new substitutions potentially associated with HCV GT4 subtype therapy failure. One limitation of this study is the small number of failing patients; however, this is inherent to the high efficacy of DAAs against GT4 HCV. More studies including a large number of HCV GT4 patients are warranted, possibly coupled to in vitro experiments, to help confirm and fully understand the role of novel substitutions in drug escape.

\section{Conclusion}

Overall, these findings confirm the importance of UDPS in the analysis of quasispecies variability, and suggest a potential role in updating the list of new substitutions potentially associated with HCV GT4 subtype therapy failure. One limitation of this study is the small number of failing patients, however, this is inherent to the high efficacy of DAAs against GT4 HCV. More studies including a larger number of HCV GT4 patients are warranted, possibly coupled to in vitro experiments, to help confirm and fully understand the role of novel substitutions in drug escape.

\section{Acknowledgment}

This study has been partly supported by grants from the Italian Ministry of Health, Ricerca Corrente.

\section{Author contributions}

$\mathrm{CM}, \mathrm{EG}, \mathrm{CT}$, and ARG designed the study. ARG, CM, MRC, and FM wrote the manuscript. MS, CM, EG, and BB analyzed the data. BB, CT, LL, SC, and GD collected the data. All authors contributed toward data analysis, drafting and revising the paper, gave final approval of the version to be published, and agree to be accountable for all aspects of the work.

\section{Disclosure}

The authors report no conflicts of interest in this work.

\section{References}

1. Gower E, Estes C, Blach S, Razavi-Shearer K, Razavi H. Global epidemiology and genotype distribution of the hepatitis $\mathrm{C}$ virus infection. J Hepatol. 2014;61(1 Suppl):S45-S57.

2. Kamal SM. Hepatitis $C$ virus genotype 4 therapy: progress and challenges. Liver Int. 2011;31(Suppl 1):45-52.

3. Kamal SM. Improving outcome in patients with hepatitis $\mathrm{C}$ virus genotype 4. Am J Gastroenterol. 2007;102(11):2582-2588.

4. Messina JP, Humphreys I, Flaxman A, et al. Global distribution and prevalence of hepatitis C virus genotypes. Hepatology. 2015;61(1):77-87.

5. Degré D, Sersté T, Lasser L, et al. Sofosbuvir in Combination with Simeprevir +/- Ribavirin in Genotype 4 Hepatitis C Patients with Advanced Fibrosis or Cirrhosis: A Real-World Experience from Belgium. PLoS One. 2017;12(1):e0170933.

6. Al Ashgar HI, Khan MQ, Al-Ahdal M, et al. Hepatitis C genotype 4 : genotypic diversity, epidemiological profile, and clinical relevance of subtypes in Saudi Arabia. Saudi J Gastroenterol. 2013;19(1):28-33.

7. Ray SC, Arthur RR, Carella A, Bukh J, Thomas DL. Genetic epidemiology of hepatitis C virus throughout Egypt. J Infect Dis. 2000;182(3):698-707.

8. Antaki N, Craxi A, Kamal S, et al. The neglected hepatitis C virus genotypes 4, 5 and 6: an international consensus report. Liver Int. 2010;30(3):342-355. 
9. Asselah T, De Muynck S, Broët P, et al. IL28B polymorphism is associated with treatment response in patients with genotype 4 chronic hepatitis C. J Hepatol. 2012;56(3):527-532.

10. Buti M, Calleja JL, Lens S, et al. Simeprevir in combination with sofosbuvir in treatment-naïve and -experienced patients with hepatitis $C$ virus genotype 4 infection: a Phase III, open-label, single-arm study (PLUTO). Aliment Pharmacol Ther. 2017;45(3):468-475.

11. Degré D, Sersté T, Lasser L, et al. Sofosbuvir in Combination with Simeprevir +/- Ribavirin in Genotype 4 Hepatitis C Patients with Advanced Fibrosis or Cirrhosis: A Real-World Experience from Belgium. PLoS One. 2017;12(1):e0170933.

12. Schnell G, Tripathi R, Beyer J, et al. Hepatitis C virus genotype 4 resistance and subtype demographic characterization of patients treated with ombitasvir plus paritaprevir/ritonavir. Antimicrob Agents Chemother. 2015;59(11):6807-6815.

13. European Association for Study of Liver. EASL Recommendations on Treatment of Hepatitis C 2015. J Hepatol. 2015;63(1):199-236.

14. Halfon P, Mohamed S, Penaranda G, et al. Hepatitis C genotype 4R resistance-associated polymorphisms: The achilles heel of the nonstructural 5A inhibitors? Hepatology. 2016;64(2):697-698.

15. Fevery B, Verbinnen T, Peeters M, et al. Virology analyses of HCV genotype 4 isolates from patients treated with simeprevir and peginterferon/ribavirin in the Phase III RESTORE study. $J$ Viral Hepat. 2017;24(1):28-36.

16. Zhou N, Hernandez D, Ueland J, et al. NS5A Sequence Heterogeneity and Mechanisms of Daclatasvir Resistance in Hepatitis C Virus Genotype 4 Infection. J Infect Dis. 2016;213(2):206-215.

17. Lindström I, Kjellin M, Palanisamy N, et al. Prevalence of polymorphisms with significant resistance to NS5A inhibitors in treatment-naive patients with hepatitis $\mathrm{C}$ virus genotypes 1a and 3a in Sweden. Infect Dis (Lond). 2015;47(8):555-562.

18. Pilon R, Leonard L, Kim J, et al. Transmission patterns of HIV and hepatitis $\mathrm{C}$ virus among networks of people who inject drugs. PLoS One. 2011;6(7):e22245.

19. Kalaghatgi P, Sikorski AM, Knops E, et al. Geno2pheno[HCV] - A Web-based Interpretation System to Support Hepatitis C Treatment Decisions in the Era of Direct-Acting Antiviral Agents. PLoS One. 2016;11(5):e0155869.

20. Fu L, Niu B, Zhu Z, Wu S, Li W. CD-HIT: accelerated for clustering the next-generation sequencing data. Bioinformatics. 2012;28(23): 3150-3152.
21. Bartolini B, Giombini E, Zaccaro P, et al. Extent of HCV NS3 protease variability and resistance-associated mutations assessed by next generation sequencing in $\mathrm{HCV}$ monoinfected and $\mathrm{HIV} / \mathrm{HCV}$ coinfected patients. Virus Res. 2013;177(2):205-208.

22. Smith DB, Bukh J, Kuiken C, et al. Expanded classification of hepatitis $\mathrm{C}$ virus into 7 genotypes and 67 subtypes: updated criteria and genotype assignment web resource. Hepatology. 2014;59(1):318-327.

23. Tamura K, Stecher G, Peterson D, Filipski A, Kumar S. MEGA6: Molecular Evolutionary Genetics Analysis version 6.0. Mol Biol Evol. 2013;30(12):2725-2729.

24. National Cancer Institute [webpage on the Internet]. Common Terminology Criteria for Adverse Events -CTCAE-. Available from: https:// ctep.cancer.gov/protocoldevelopment/electronic_applications/ctc. htm\#ctc_50. Accessed April 10, 2018.

25. McPhee F, Hernandez D, Zhou N, et al. Virological escape in HCV genotype-1-infected patients receiving daclatasvir plus ribavirin and peginterferon alfa-2a or alfa-2b. Antivir Ther. 2014;19(5):479-490.

26. Roulot D, Thibault V, Laforest C, et al; ANRS HC32 QUATTRO study group. Efficacy of daclatasvir-based quadruple therapy in nonresponder patients infected by hepatitis $\mathrm{C}$ virus genotype 4: the ANRS HC32 QUATTRO study. Eur J Gastroenterol Hepatol. 2018;30(3):302-309.

27. Nelson DR, Cooper JN, Lalezari JP, et al; ALLY-3 Study Team. Alloral 12-week treatment with daclatasvir plus sofosbuvir in patients with hepatitis $\mathrm{C}$ virus genotype 3 infection: ALLY-3 phase III study. Hepatology. 2015;61(4):1127-1135.

28. Poordad F, Schiff ER, Vierling JM, et al. Daclatasvir with sofosbuvir and ribavirin for hepatitis $\mathrm{C}$ virus infection with advanced cirrhosis or postliver transplantation recurrence. Hepatology. 2016;63(5):1493-1505.

29. Wyles DL, Ruane PJ, Sulkowski MS, et al; ALLY-2 Investigators. Daclatasvir plus Sofosbuvir for HCV in Patients Coinfected with HIV-1. N Engl J Med. 2015;373(8):714-725.

30. Lok AS, Gardiner DF, Lawitz E, et al. Preliminary study of two antiviral agents for hepatitis C genotype 1. NEngl J Med. 2012;366(3):216-224.

31. Suzuki Y, Ikeda K, Suzuki F, et al. Dual oral therapy with daclatasvir and asunaprevir for patients with HCV genotype $1 \mathrm{~b}$ infection and limited treatment options. J Hepatol. 2013;58(4):655-662.

32. Sorbo MC, Cento V, Di Maio VC, et al. Hepatitis C virus drug resistance associated substitutions and their clinical relevance: Update 2018. Drug Resist Updat. 2018;37:17-39.

33. Toshikuni N. Therapy with Direct-Acting Antiviral Agents for Hepatitis C-Related Liver Cirrhosis. Gut Liver. 2017;11(3):335-348. 


\section{Supplementary material}

Table SI Primers used to detect NS5A and NS5B regions of HCV

\begin{tabular}{|c|c|c|c|c|c|c|}
\hline Target & $\begin{array}{l}\text { Amplification } \\
\text { step }\end{array}$ & Primer & Primer sequence $5^{\prime}-3^{\prime}$ & $\begin{array}{l}\text { Position on } \\
\text { DQ4 I } 8786 \\
\text { (GT4d) }\end{array}$ & $\begin{array}{l}\text { Fragment } \\
\text { length (bp) }\end{array}$ & Reference \\
\hline NS5A & $\begin{array}{l}\text { First round } \\
\text { Second round }\end{array}$ & $\begin{array}{l}\text { NS5A_out.for } \\
\text { NS5A_out.rev } \\
\text { NS5A_in.for } \\
\text { NS5A_in.rev }\end{array}$ & $\begin{array}{l}\text { GGGCDGTRCARTGGATGAAC } \\
\text { GGMTCGAADGAGTCMAGAAT } \\
\text { GATGAACMGGCTSATMGCSTTCG } \\
\text { CCCRTCCAMTTCWGTGAARAAYTC }\end{array}$ & $\begin{array}{l}5963-5982 \\
6992-6973 \\
5976-5998 \\
6612-6589\end{array}$ & $\begin{array}{l}1,030 \\
637\end{array}$ & 17 \\
\hline NS5B & $\begin{array}{l}\text { First round } \\
\text { Second round }\end{array}$ & $\begin{array}{l}\text { NS5B_out.for } \\
\text { NS5B_out.rev } \\
\text { NS5B_in.for } \\
\text { NS5B_in.rev }\end{array}$ & $\begin{array}{l}\text { GTSTGGIARGACYTICTGGAAGAC } \\
\text { RGIGCRGARTACCTRGTCATAGCCT } \\
\text { IACYATCATGGCIAARARYGAGGT } \\
\text { ACCTRGTCATAGCCTCCGTGAA }\end{array}$ & $\begin{array}{l}7846-7869 \\
8528-8504 \\
7890-7913 \\
8518-8497\end{array}$ & $\begin{array}{l}683 \\
629\end{array}$ & 18 \\
\hline
\end{tabular}

Infection and Drug Resistance is an international, peer-reviewed openaccess journal that focuses on the optimal treatment of infection (bacterial, fungal and viral) and the development and institution of preventive strategies to minimize the development and spread of resistance. The journal is specifically concerned with the epidemiology of antibiotic resistance and the mechanisms of resistance development and diffusion in both hospitals and the community. The manuscript management system is completely online and includes a very quick and fair peerreview system, which is all easy to use. Visit http://www.dovepress.com/ testimonials.php to read real quotes from published authors.

Submit your manuscript here: https://www.dovepress.com/infection-and-drug-resistance-journal 